\title{
Line-field confocal optical coherence tomography for three- dimensional skin imaging
}

\author{
Jonas Ogien ${ }^{\mathrm{a}}$, Anthony Daures ${ }^{\mathrm{a}}$, Maxime Cazalas ${ }^{\mathrm{a}}$, Jean-Luc Perrot ${ }^{\mathrm{b}}$, Arnaud Dubois $^{\mathrm{c}}$

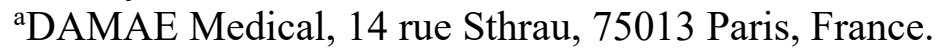 \\ bervice dermatologie, CHU St-Etienne, 42055 Saint-Etienne, France. \\ 'Université Paris-Saclay, Institut d'Optique Graduate School, CNRS, Laboratoire Charles Fabry, \\ 91127 Palaiseau, France.
}

\begin{abstract}
This paper reports on the latest advances in line-field confocal optical coherence tomography (LC-OCT), a recently invented imaging technology that now allows the generation of either horizontal $(x \times y)$ section images at an adjustable depth or vertical $(\mathrm{x} \times \mathrm{z})$ section images at an adjustable lateral position, as well as three-dimensional images. For both two-dimensional imaging modes, images are acquired in real-time, with real-time control of the depth and lateral positions. Three-dimensional $\left(x^{\times} \times y \times z\right)$ images are acquired from a stack of horizontal section images. The device is in the form of a portable probe. The handle of the probe has a button and a scroll wheel allowing the user to control the imaging modes. Using a supercontinuum laser as a broadband light source and a high numerical microscope objective, an isotropic spatial resolution of $\sim 1 \mu \mathrm{m}$ is achieved. The field of view of the three-dimensional images is $1.2 \times 0.5 \times 0.5 \mathrm{~mm}^{3}(\mathrm{x} \times \mathrm{y} \times \mathrm{z})$. Images of skin tissues are presented to demonstrate the potential of the technology in dermatology.
\end{abstract}

Keywords: Optical coherence tomography, microscopy, three-dimensional imaging, dermatology.

\section{Introduction}

Optical coherence tomography (OCT) is a technology based on low-coherence optical interferometry for imaging biological tissues with micrometer-scale spatial resolution [1,2]. OCT is commonly used in several medical fields [3], especially in ophthalmology to obtain images of the retina and the anterior segment of the eye [4]. OCT has begun to be used in interventional cardiology [5], and in gastroenterology for the detection and diagnosis of tumors [6,7]. OCT can be a useful tool for noninvasive imaging of brain tissues $[8,9]$. OCT also shows promise in dermatology to improve the diagnosis process of skin lesions [10].

In the field of dermatology, high spatial resolution imaging is necessary to resolve subtle morphological changes in skin tissues resulting from the early progression of lesions. Since the introduction of OCT about 30 years ago, significant progress has been achieved in the spatial resolution of OCT images. The axial resolution in OCT is governed by the temporal coherence of the illumination light source [2]. Improvement in the axial resolution of OCT images has been achieved through the emergence of efficient broadband light sources. Axial resolutions down to $\sim 1 \mu \mathrm{m}$ have been achieved with mode-locked lasers [11] and more recently with supercontinuum lasers [12-14]. The lateral resolution of the OCT images depends on how the light beam is focused on the sample [2]. Depending on whether the signal is acquired as a function of the optical frequency (in frequency/Fourier-domain OCT, referred to as FD-OCT) or as a function of time (in time-domain OCT, referred to as TD-OCT), the beam focusing constraints differ, which has an impact on the lateral resolution.

In TD-OCT, the reflectivity profile of the sample as a function of depth (an A-scan) is acquired by scanning the sample depth [1,2]. In FD-OCT, the reflectivity profile of the sample as a function of depth is acquired without scanning the depth, by measuring the spectrum of the interferometric signal [2]. In both TD-OCT and FD-OCT, a B-scan (a vertical section image) is then obtained by lateral scanning of the light beam to acquire several adjacent A-scans. En face images can also be obtained by scanning the beam in two lateral directions. En face imaging has been implemented in both TD-OCT and FD-OCT [15]. 
FD-OCT is superior to TD-OCT in terms of acquisition rate and detection sensitivity [16], but has shortcomings including a limited lateral resolution [17]. Since all points in the depth range in the sample must be in focus simultaneously, a depth of field (DOF) at least equal to the depth range is needed, which limits the beam focusing. Several approaches have been reported to try to circumvent this limitation, i.e. improve the lateral resolution without sacrificing the imaging depth. One approach consists in increasing the DOF using Bessel beams produced by axicon lenses [17-20] or coaxially focused multimode beams [20] or apodized beams $[21,22]$. Computational methods have also been reported, such as interferometric synthetic aperture microscopy $[23,24]$ or digital refocusing [25,26]. Another approach consists in overlapping and fusing several B-scans acquired at different depths by using a phase plate [27] or multiple light beams focused at different depths, in order to image over a depth range larger than the DOF [28,29]. This approach has also been combined with Bessel beam illumination [30], or using several beams with different wavelengths associated to different depths [31,32]. The B-scans to combine can also be obtained using a single beam refocused at different depths, a method referred to as C-mode scanning [33] or Gabordomain OCT $[34,35]$. Let us note that this approach has also been combined with digital refocusing to improve its throughput [36].

Unlike FD-OCT, TD-OCT offers the possibility to continuously adjust the focus as a function of depth, making TD-OCT more suitable for producing images with high lateral resolution. Dynamic focus tracking in TD-OCT with free-space optics has been reported, but the tracking rate was slow [37,38]. A microelectromechanical systems (MEMS) mirror was designed for high-speed dynamic focus tracking, but without demonstration of the imaging capability in vivo [39]. Another approach consists of acquiring a sequence of images by gradually shifting the focus onto the sample and then fusing together the in-focus imaging zones [40]. This process results in a trade-off between lateral resolution and image acquisition speed. Another method is to collect multiple foci simultaneously with a multifocus fiber tip array [41]. Despite these advances, however, high lateral resolution TD-OCT imaging using dynamic focusing remains difficult since a high tracking speed is required.

Line-field confocal optical coherence tomography (LC-OCT) is a recently invented imaging technology that can produce images without the lateral resolution limitation of FD-OCT and without the speed limitation of conventional dynamically-focused TD-OCT. This paper reports on the latest LC-OCT prototype. Compared to the previous prototype capable of acquiring either horizontal (en face, $\mathrm{x} \times \mathrm{y}$ ) section images at an adjustable depth or vertical section images (B-scan, $\mathrm{x} \times \mathrm{z}$ ) at an adjustable lateral position [42], three-dimensional images $(x \times y \times z)$ can now be acquired. The technical characteristics of the different imaging modes are described. The device is now in the form of a portable probe. The handle of the probe has a button and a scroll wheel allowing the user to control the imaging modes. Three-dimensional images of skin tissues are presented to demonstrate the potential of LC-OCT in dermatology.

\section{The LC-OCT technique}

\subsection{General principle and interest}

Line-field confocal optical coherence tomography (LC-OCT) is an imaging technique based on TD-OCT with illumination of the sample with a line of light and detection using a line camera [42-47]. This differs from conventional TD-OCT where the sample is illuminated point by point and a vertical section image is obtained from several A-scans acquired sequentially. In conventional TD-OCT, the scan of the sample depth is repeated after each A-scan acquisition, which requires depth scanning at high speed to obtain an image in real-time. In LC-OCT, all the A-scans of a vertical section image are acquired in parallel. The speed of the depth scan in LC-OCT can therefore be reduced compared to point-scanning TD-OCT, without increasing the image acquisition time. Producing a vertical section image in real time with LC-OCT requires scanning the sample depth at a frequency of a few Hertz only. Obtaining a vertical section image at the same frame rate in point-scanning TD-OCT requires depth scanning at a frequency increased by a factor equal to the number of A-scans (factor of 2048 compared to LC-OCT using a line camera with 2048 pixels). The significantly slower depth scan in LC-OCT makes it possible to dynamically focus a microscope objective at a speed suitable for the real-time acquisition of high-resolution vertical section images. In LC-OCT, vertical section images can thus be obtained without the lateral resolution limitation of FD-OCT and without the speed limitation of conventional dynamically-focused TD-OCT. In addition, with the recent LC-OCT prototypes, it is also possible to obtain high-resolution horizontal section images by scanning the illumination line laterally on the sample [47]. 


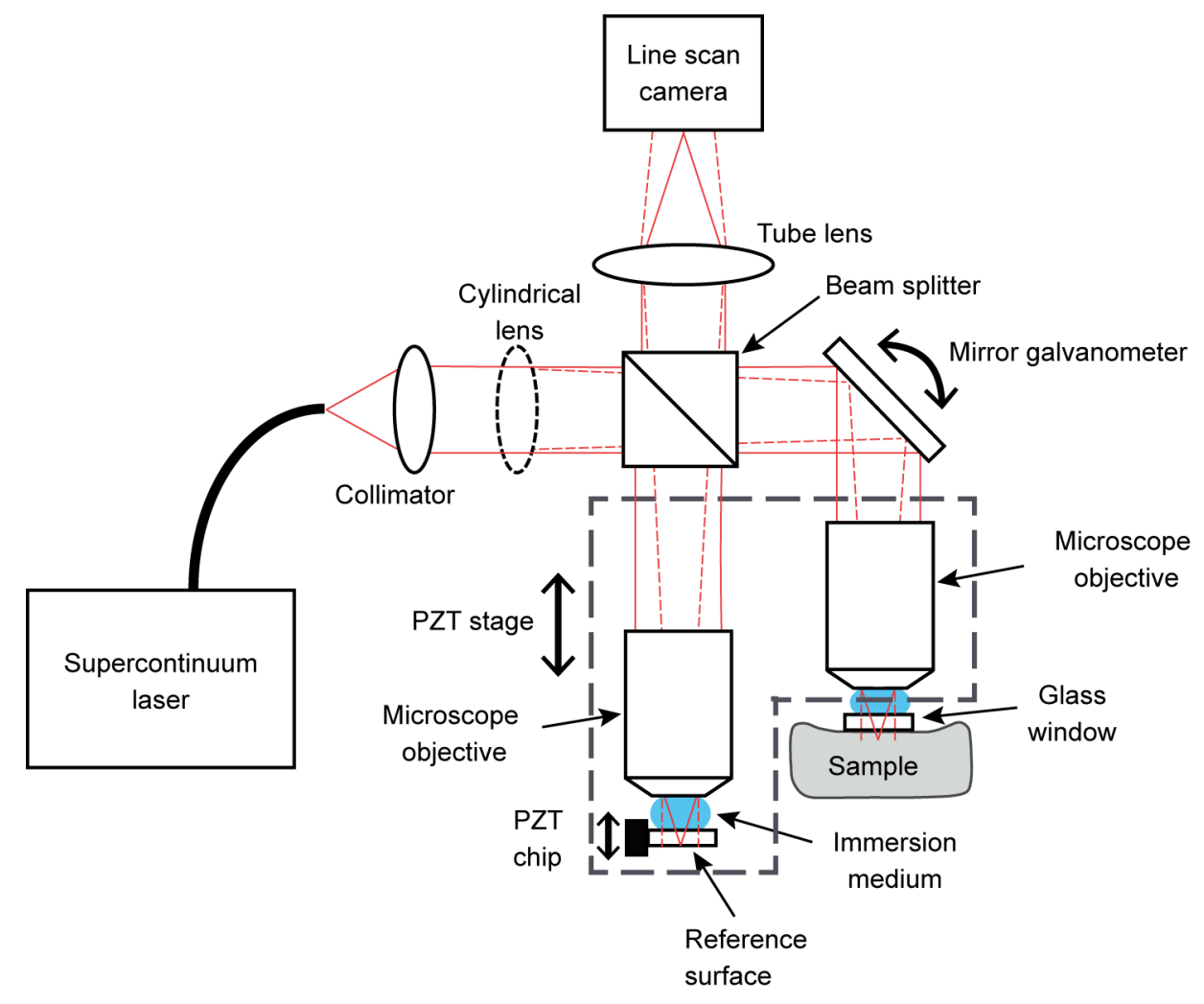

Figure 1. Schematic diagram of the latest LC-OCT prototype.

\subsection{Latest prototype}

The experimental setup of the latest LC-OCT prototype is shown schematically in Figure 1. It is based on a Linnik-type interferometer with a piezoelectric (PZT) stage for depth (z) scanning and a mirror galvanometer for lateral (y) scanning. The reference surface of the interferometer is mounted on a piezoelectric (PZT) chip that can oscillate to generate a phase modulation. A supercontinuum laser is used as a broadband light source at a detected central wavelength of about $750 \mathrm{~nm}$. A cylindrical lens is employed to generate a line of light that is focused on the sample. The image of this line is projected on a line-scan camera. All the elements used for illumination and imaging (laser, optics, camera) are the same as those of the previously reported LC-OCT system [47]. The microscope objectives are also the same (20x, numerical aperture of 0.5). Silicone oil is used as an immersion medium with a refractive index of 1.4, close to the mean refractive index of human skin. Glass plates are placed in both arms of the interferometer under the microscope objectives for subject stabilization and for providing a low reflectivity $(3.5 \%)$ reference surface. The whole reference arm of the interferometer and the microscope objective in the sample arm (elements in the dashed frame in Figure 1) are mounted on the piezoelectric stage for vertical section imaging. The general operation of the device and the image display are controlled by a new $\mathrm{C}++$ software. The device is now in the form of a portable probe (see Figure 2). The dimensions of the probe are $215 \mathrm{~mm}$ (length) $\times 120$ $\mathrm{mm}$ (width) $\times 208 \mathrm{~mm}$ (height) and the weight is $1.2 \mathrm{~kg}$. A button on the probe handle allows the user to record an image in both real-time imaging modes (vertical or horizontal). A double-click on the button enables to switch from one real-time imaging mode to another. The probe handle is also equipped with a scroll wheel, which allows the user to adjust the lateral/depth positions in the horizontal/vertical real-time imaging modes. When clicked, the scroll wheel also allows the user to choose between video recording and three-dimensional acquisition. 


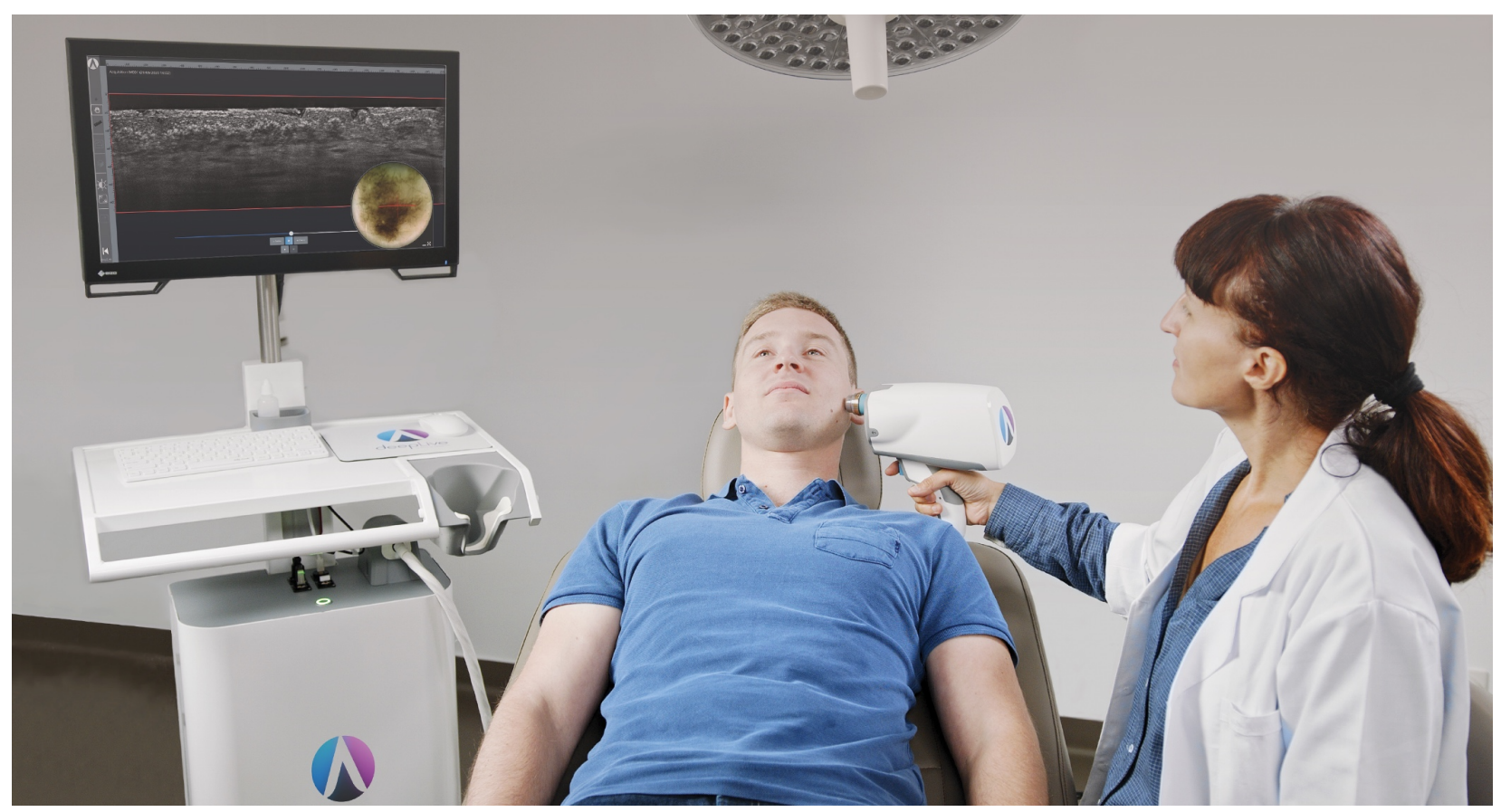

Figure 2. LC-OCT probe and cart used by a dermatologist in a clinical setting.

\section{The three imaging modes}

Compared to the previous LC-OCT prototype capable of acquiring either horizontal (en face, $\mathrm{x} \times \mathrm{y}$ ) section images at an adjustable depth or vertical section images (B-scan, $\mathrm{x} \times \mathrm{z}$ ) at an adjustable lateral position [42], three-dimensional images $(x \times y \times z)$ can now be acquired with the new device. The operating principle and the technical characteristics of the three imaging modes are described below.

\subsection{Vertical section imaging}

A vertical section image is acquired by activating the PZT stage to scan the sample depth. The displacement of the PZT stage is analogically controlled by a custom electronic board that sends a periodic driving current (see Figure 3). The PZT oscillates at a frequency of $\mathrm{f}_{\mathrm{PZT}}=8 \mathrm{~Hz}$ according to asymetrical sawthooths. The amplitude of the oscillation is $500 \mu \mathrm{m}$, but due to the non-linearity of the PZT stage displacement at the edges of the sawtooths, the images are acquired over only $80 \%$ of the total amplitude of the oscillation. The vertical section images are thus acquired over an effective depth range of $Z=400 \mu \mathrm{m}$. The camera is synchronized with the oscillation of the PZT. The camera frame rate is set at $70 \mathrm{kHz}$, so that the step between two consecutive lines corresponds to a displacement of the PZT of $\delta=71 \mathrm{~nm}$, i.e. to a phase shift of $\pi / 2$. A stack of $\mathrm{Z} / \delta=5,600$ lines is acquired during each positive slope of the depth scan. A vertical section image is obtained by processing the acquired stack using a five-frame fringe envelope detection algorithm [48]. Acquisition and processing of stacks is repeated continuously during the round-trips of the PZT stage. The images are displayed in real-time at 8 frames/s in logarithmic scale with auto-adjusted contrast after being appropriately rescaled. The size of each vertical section image is $2048 \times 680$ pixels $(\mathrm{x} \times \mathrm{z})$, corresponding to a field of view of $1.2 \mathrm{~mm} \times 0.4 \mathrm{~mm}(\mathrm{x} \times \mathrm{z})$.

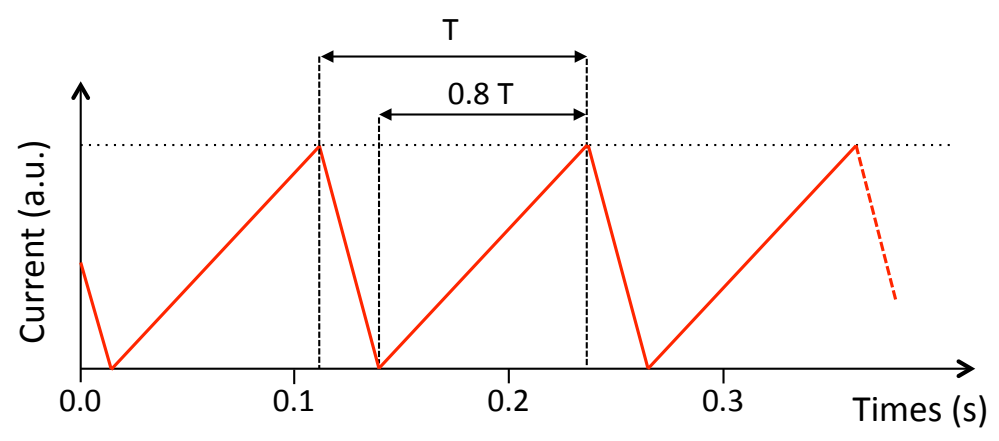


Figure 3. Periodic current driving the oscillation of the PZT stage. The asymmetric triangle signal has a frequency of $\mathrm{f}_{\mathrm{PZT}}=1 / \mathrm{T}=8 \mathrm{~Hz}$ and a duty cycle of $80 \%$. Images are acquired only during the slow positive ramps.

In the vertical section imaging mode, the scroll wheel on the probe handle allows the user to modify the value of a direct current sent to the mirror galvanometer by the electronic board. This enables to laterally navigate within the sample being imaged, in real-time. The accuracy of the lateral position is determined by the noise in the rotation of the mirror galvanometer. In practice, the position of the laser line focused on the sample does not fluctuate more than the lateral resolution of the imaging system. Therefore, the positioning accuracy can be considered to be better than $1 \mu \mathrm{m}$. This is of course a much higher positioning resolution than can be achieved by moving the probe manually.

\subsection{Horizontal section imaging}

In the horizontal section imaging mode, the mirror galvanometer oscillates according to asymmetrical sawtooths, at a frequency $\mathrm{f}_{\text {galvo }}=8 \mathrm{~Hz}$ (see Figure 4) to scan the illumination line on the sample over a field of $500 \mu \mathrm{m}$. Let us note that the scanning field of $500 \mu \mathrm{m}$ represents $95 \%$ of the total scanning amplitude of the mirror galvanometer, to avoid non-linearities at the edges of the sawtooths. The mirror galvanometer is analogically controlled by the same custom electronic board as the PZT stage. The camera frame rate is set to $\mathrm{f}_{\text {cam }}=100 \mathrm{kHz}$. Two consecutive lines acquired by the camera are thus separated by a lateral distance of $47 \mathrm{~nm}$. A stack of 10,600 lines is acquired during each positive slope of the lateral scan. The reference surface of the interferometer oscillates sinusoidally to generate a phase modulation. The current driving the oscillation is generated by the same custom electronic board that controls the PZT stage and mirror galvanometer. It is amplified before being sent to the PZT chip. A sinusoidal phase-shifting algorithm is used to calculate each line of the horizontal section image from an algebraic combination of five consecutive lines acquired by the camera [48]. The frequency of the phase modulation and its amplitude are set empirically at $8 \mathrm{kHz}$ and $1 \mu \mathrm{m}$ respectively to optimize the image quality. Acquisition and processing is repeated continuously during the round-trips of the mirror galvanometer. The horizontal section images are displayed in real-time at 8 frames/s in logarithmic scale with auto-adjusted contrast after being appropriately rescaled. The size of each horizontal section image is $2048 \times 850$ pixels $(\mathrm{x} \times \mathrm{y})$, corresponding to a field of view of $1.2 \mathrm{~mm} \times 0.5 \mathrm{~mm}(\mathrm{x} \times \mathrm{y})$.

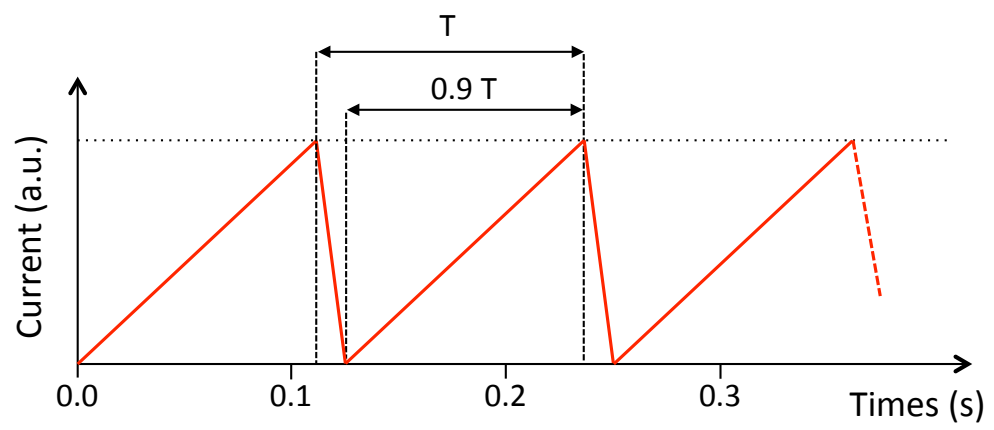

Figure 4. Periodic current driving the oscillation of the mirror galvanometer. The asymmetric triangle signal has a frequency of $\mathrm{f}_{\text {galvo }}=1 / \mathrm{T}=8 \mathrm{~Hz}$ and a duty cycle of $90 \%$. Images are acquired only during the slow positive ramps.

In the horizontal section imaging mode, the scroll wheel on the probe handle allows the user to control the value of a direct current sent to the PZT stage by the electronic board. This makes it possible to control at what depth the sample is imaged, between 0 and $500 \mu \mathrm{m}$, in real time and with an accuracy determined by the resolution of the PZT stage which is much better than the axial resolution of the imaging system of $\sim$ $1 \mu \mathrm{m}$.

\subsection{Three-dimensional imaging}

Three-dimensional images can be produced from a stack of horizontal section images acquired at successive depths. In the three-dimensional imaging mode, horizontal section images are continuously acquired while a ramp signal is sent by the electronic board to the PZT stage to displace it from 0 to $500 \mu \mathrm{m}$. The ramp 
signal is adjusted so that successive horizontal section images correspond to a depth difference of one micrometer, approximately equal to the axial resolution of the imaging system. This results in threedimensional stacks of $2048 \times 850 \times 500$ pixels, scaled to $1200 \times 500 \times 500$ pixels for proper aspect ratio. The three-dimensional images correspond to a volume of up to $1.2 \times 0.5 \times 0.5 \mathrm{~mm}^{3}(\mathrm{x} \times \mathrm{y} \times \mathrm{z})$.

Since the PZT stage moves continuously during the lateral scan with the mirror galvanometer, the images of a stack are not perfectly horizontal. However, since the depth difference between the edges of each image of a stack is one micrometer, the tilt is imperceptible. Let us note that the implementation of this acquisition scheme (continuous imaging over a continuous ramp) is simple, as it requires no particular synchronization between the PZT stage and the mirror galvanometer.

In practice, three-dimensional imaging is decided by the user by clicking the scroll wheel of the probe handle and turning it to the appropriate direction. One direction of rotation initiates the acquisition of a threedimensional image, while the other direction initiates a video recording of the current imaging mode. The effect of rotating the scroll wheel back and forward is shown on the software display window when the user clicks on the scroll wheel. Once a three-dimensional acquisition has started, the PZT stage and the mirror galvanometer move continuously until the PZT stage reaches its maximum depth of $500 \mu \mathrm{m}$. When the maximum depth is reached, the horizontal image acquisition stops and the system returns to the imaging mode it was in before the three-dimensional acquisition was performed. In the three-dimensional imaging mode, the acquired horizontal images are displayed and the user can stop the acquisition whenever he wants, without waiting for the PZT stage to reach its maximum depth, by clicking on the scroll wheel or on the button on the probe handle. In that case, the depth of the acquired volumetric image is less than $500 \mu \mathrm{m}$ and depends on when the user stopped the acquisition.

\section{Skin imaging}

LC-OCT has previously been shown to yield high quality imaging of human skin, in vivo, giving access non-invasively to a level of morphological detail close to the one of histology using similar microscope objectives $(20 \mathrm{x}, \mathrm{NA}=0.5)$ [43, 49-60]. The latest LC-OCT prototype introduced here was also designed specifically for in vivo skin imaging. The skin is gently pressed again the glass window in the sample arm of the interferometer in order to hold it steady, flatten it, and maintain it at the position corresponding to the working distance of the microscope objective. A drop of paraffin oil is placed between the skin and the glass window in order to minimize parasitic reflections at the interfaces.

Figure 5 illustrates the performance of the LC-OCT prototype for the two real-time imaging modes. The images were obtained from healthy skin of a 30-year-old man. Three horizontal section images are shown for three different depths corresponding to different layers of the skin (stratum spinosum, stratum basale, superficial dermis). In the stratum spinosum (Figure 5a), the dark spots correspond to the nuclei of keratinocyte cells. Melanin, which appears as bright spots, is well resolved in the stratum basale (Figure 5b), along with keratinocyte cells presenting a higher density than in the stratum spinosum, with a smaller size. The network of fibers formed by collagen and elastin within the dermis is also visible (Figure 5c), with the possibility to identify single fibers in some regions. A vertical section image is also shown (Figure 5d), where the different layers of the skin can be identified, in particular the epidermis (including the stratum corneum) and the dermis, and the dermal-epidermal junction which separates them (which appears dark). Nuclei of keratinocyte cells are well resolved as they were in the horizontal mode. Within the dermis, blood vessels, which appear dark, can be identified. 


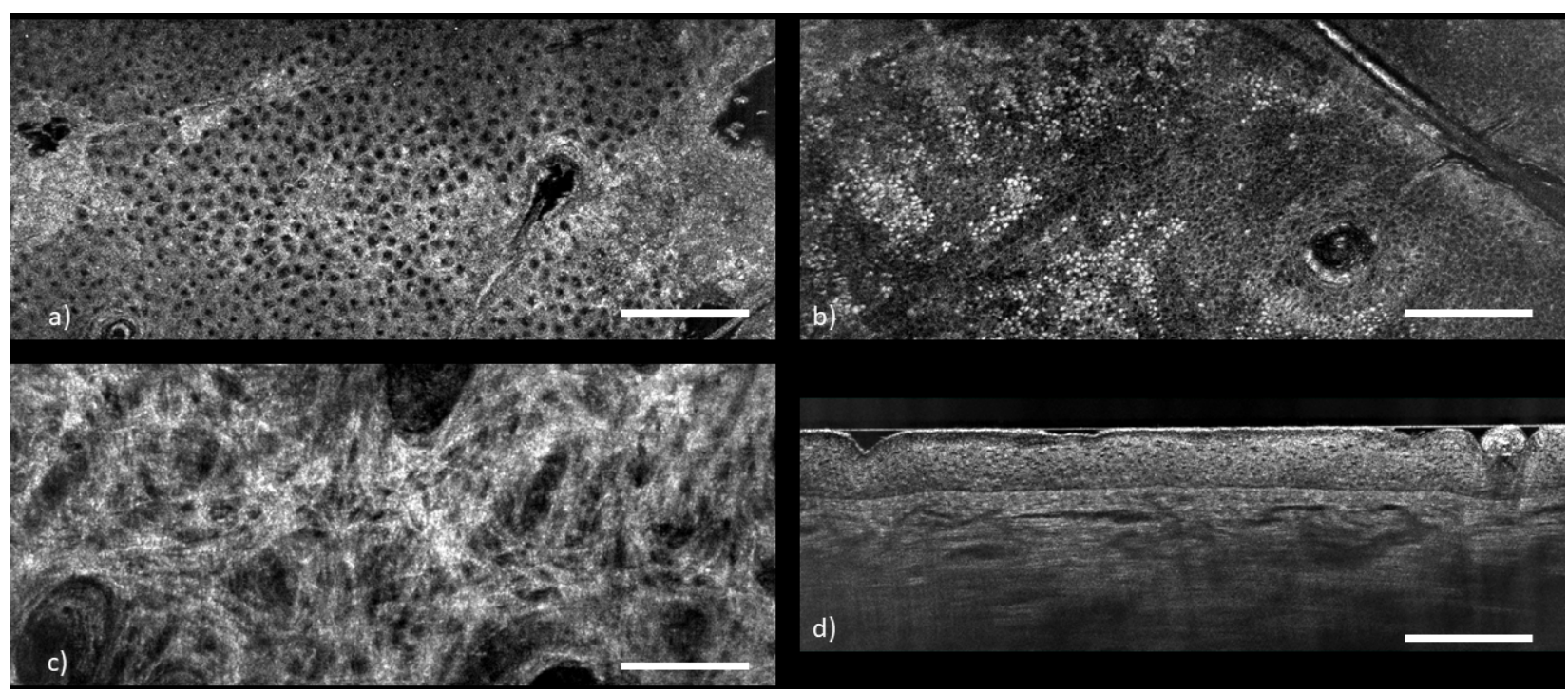

Figure 5. LC-OCT images of healthy human skin in vivo obtained in real-time ( 8 frames per second). Horizontal section images obtained at the level of the stratum spinosum (a), the stratum basale (b) and the superficial dermis (c). A vertical section image is also displayed (d). Scale bars are $200 \mu \mathrm{m}$.

The main novelty of the prototype is to be able to acquire three-dimensional images of skin, in vivo, once a region of interest has been identified by examining it using the real-time horizontal and vertical imaging modes. Owing to the quasi-isotropic resolution of LC-OCT close to $1 \mu \mathrm{m}$, the volume rendering images exhibit morphological information at cellular level in three-dimensions, and can be regarded as "histological blocks", revealing morphological features inaccessible in conventional histology where only slices in two dimensions can be analyzed.

Figure 6 shows several views of a LC-OCT volume rendering image of in vivo healthy skin, acquired on the skin of a 30-year-old man. Three-dimensional visualization was obtained using the volume rendering module of the open-source software 3D Slicer (http://www.slicer.org/). The features that were visible in the horizontal and vertical images can now be visualized in three dimensions, including for instance the skin layers and the dermal-epidermal junction, the nuclei of keratinocyte cells, elastin and collagen fibers, and blood vessels. 


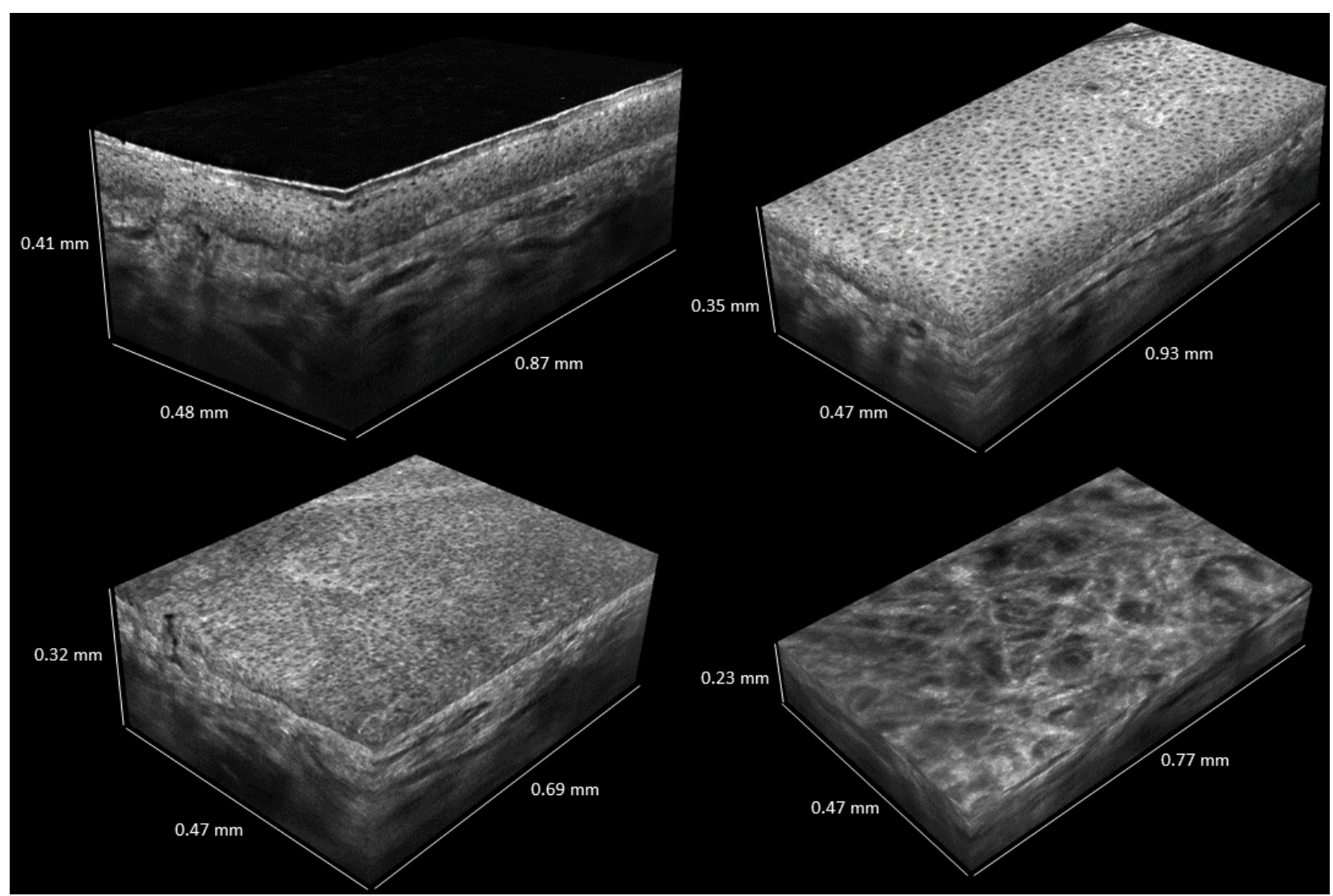

Figure 6. Three-dimensional volume rendering of in vivo human healthy skin, digitally "cut" at different depths.

Three-dimensional imaging offers several valuable ways to visualize the data for analysis. It is possible to reslice the volume along any plane of interest. In particular, it is possible to obtain vertical and horizontal images from the volume, similar to the images obtained during real-time acquisition, as shown in Figure 7a. It is also possible to navigate within the volume while visualizing such vertical and horizontal images, as shown in Figure $7 \mathrm{~b}$. 


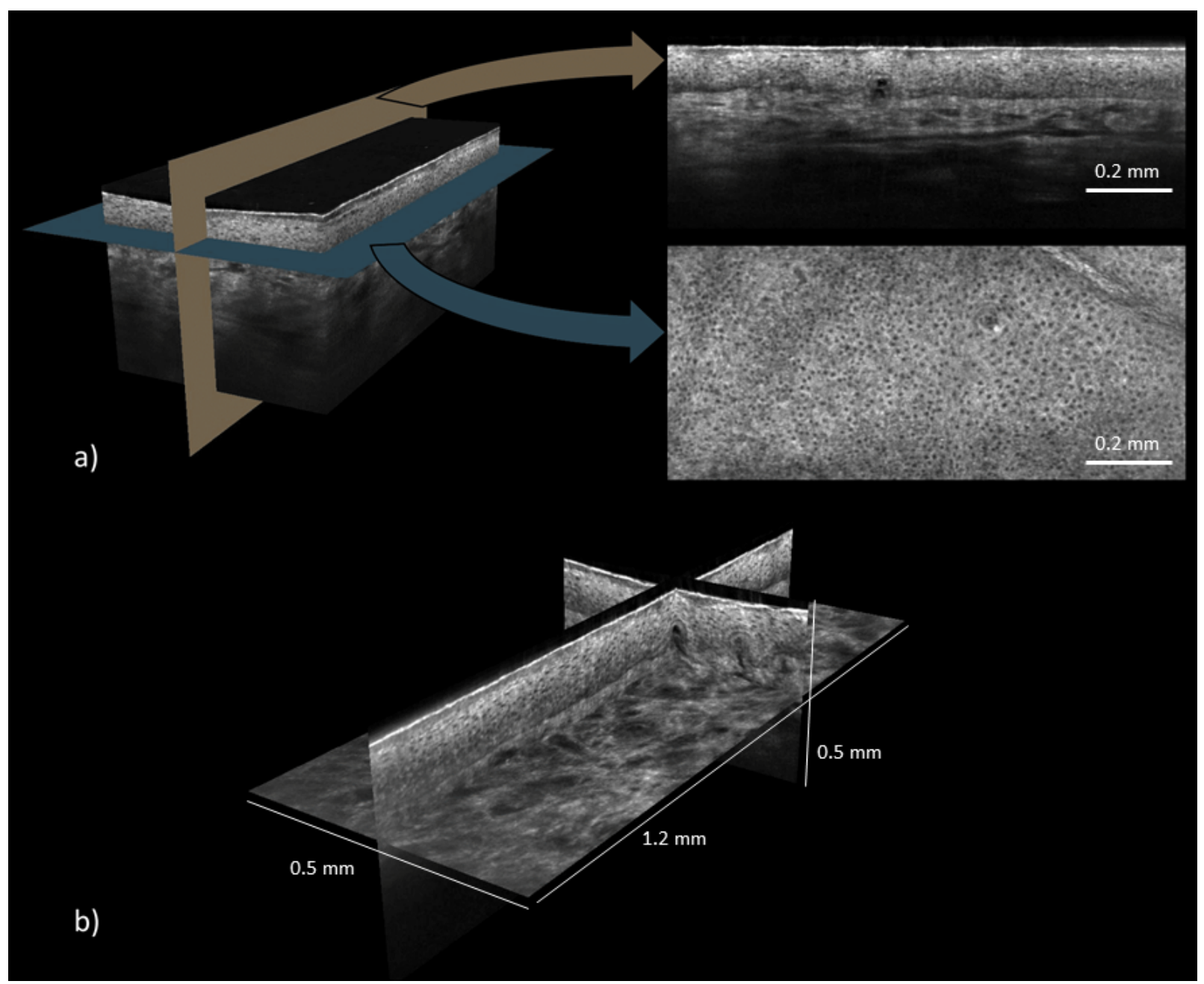

Figure 7. a) reslicing of a vertical and a horizontal image from a LC-OCT three-dimensional volume. b) Visualization of a three-dimensional volume from horizontal and vertical slices.

Other types of visualizations can be useful to analyze given features in three-dimension within the skin. Figure 8 shows for example a three-dimensional maximum intensity projection applied to a sub-region of a volume obtained in vivo on the palm of the hand, a site where the stratum corneum is very thick, yielding helicoidal sweat ducts. This technique consists in displaying the brightest voxel value encountered in each projection line of the rendered volume. As the sweat ducts are hyperreflective, the maximum intensity projection enables to clearly visualize them in three-dimensions.

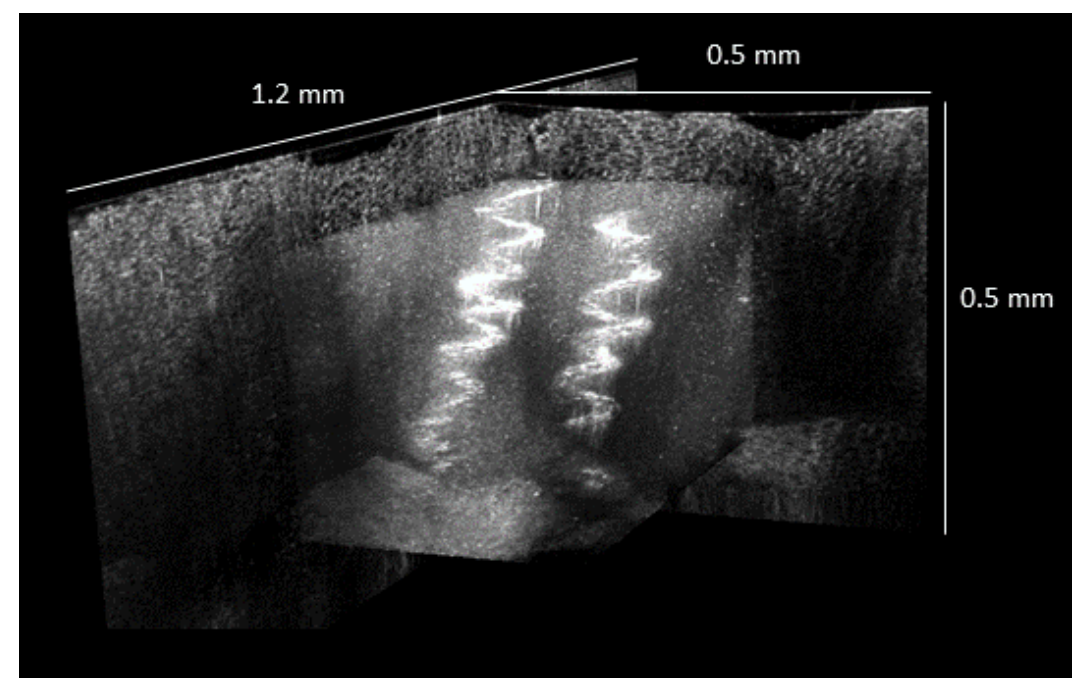

Figure 8. Three-dimensional visualization of the sweat ducts of the palm of the hand from application of a threedimensional maximum intensity projection to a LC-OCT volume. 
Conversely, a minimum intensity projection exhibits the three-dimensional aspect of hyporeflective structures, by displaying the darkest voxel value encountered in each projection line of the rendered volume. In particular, blood vessels can be visualized using this type of projection. Figure 9 shows for example a three-dimensional minimum intensity projection applied to a sub-region of a volume obtained in vivo at the level of the nailfold. Several well organized long looped capillaries can be observed. The analysis (capillaroscopy) of the capillaries in the region of the nailfold can be useful for the diagnosis of several diseases [61]. The application of a minimum intensity projection to the LC-OCT volumetric images enables to visualize those capillaries in three-dimensions, which is not feasible using conventional capillaroscopy methods.

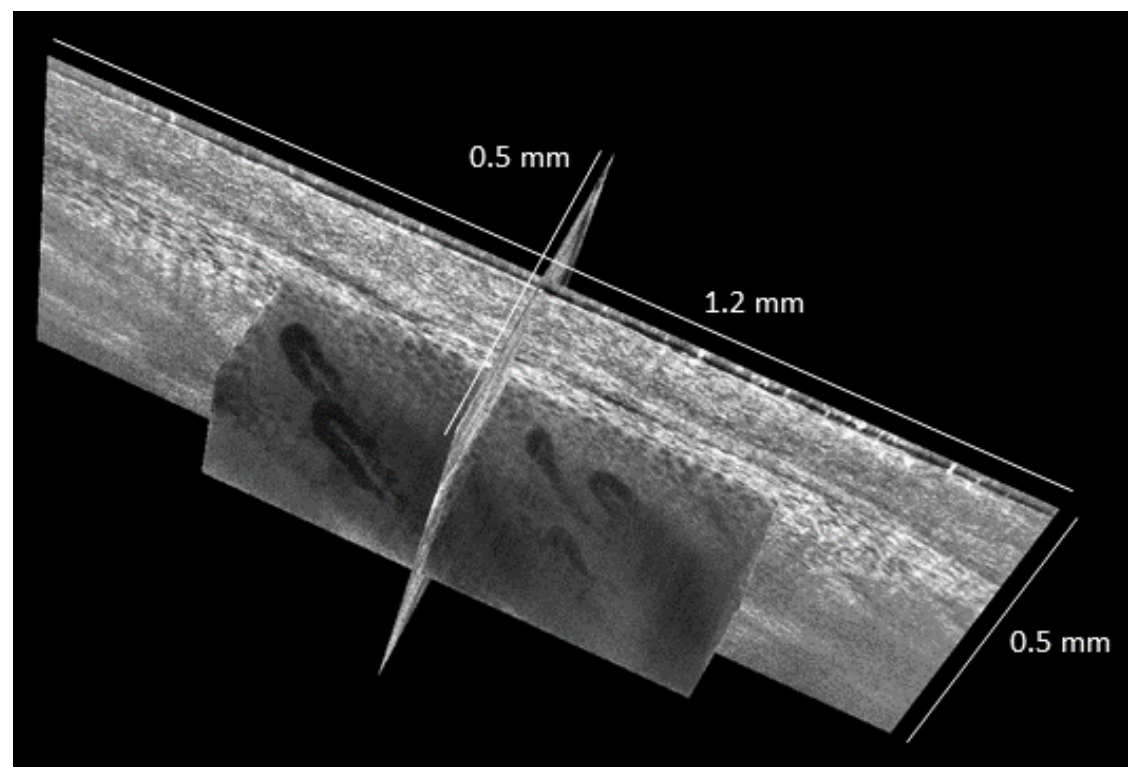

Figure 9. Three-dimensional visualization of the capillaries of the nailfold from application of a three-dimensional minimum intensity projection to a LC-OCT volume.

Eventually, let us note that three-dimensional cellular level imaging opens the way for more relevant quantification metrics of the skin, as the metrics can be obtained from full volumes. This includes for instance the keratinocyte cells or melanin distribution and density within the epidermis, or the quantification of the dermal-epidermal junction and of the epidermis thickness, which can be obtained from the segmentation of the epidermis and dermis from a three-dimensional image, as shown in Figure 10. The three-dimensional visualization of the segmentation was obtained using the segmentation module of 3D Slicer, applied to a volume previously segmented by hand using the open-source software ImageJ (https://imagej.nih.gov/ij/). 


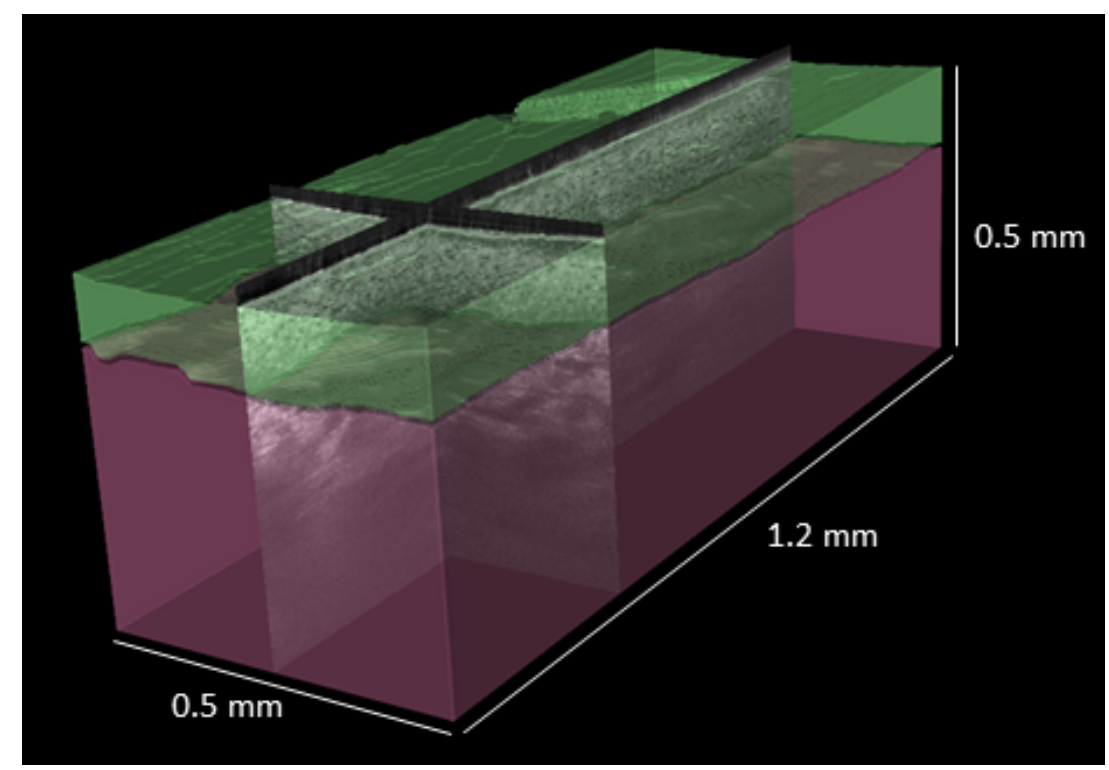

Figure 10. Three-dimensional skin layers segmentation of a LC-OCT volume. The green volume corresponds to the segmented epidermis, while the purple volume corresponds to the dermis.

\section{Conclusion}

A new LC-OCT prototype has been developed to generate both vertical section images and horizontal section images, as well as three-dimensional images. The device is in the form of a portable probe. The handle of the probe has a button and a scroll wheel for an easy control of the imaging modes. The vertical section images have a field of view of $1.2 \times 0.4 \mathrm{~mm}^{2}(\mathrm{x} \times \mathrm{z})$ and the horizontal section images have a field of view of $1.2 \times 0.5 \mathrm{~mm}^{2}(\mathrm{x} \times \mathrm{y})$. The images have a quasi-isotropic spatial resolution of $\sim 1 \mu \mathrm{m}$. They are acquired and displayed at a frame rate of $8 \mathrm{~Hz}$ in both modes. The three-dimensional images are acquired from a stack of horizontal section images acquired down to a depth of $0.5 \mathrm{~mm}$. They correspond to a volume of up to $1.2 \times 0.5 \times 0.5 \mathrm{~mm}^{3}(\mathrm{x} \times \mathrm{y} \times \mathrm{z})$.

In vivo imaging of human skin at the cellular level was demonstrated in both vertical and horizontal imaging modes. Three-dimensional cellular level imaging of human skin, in vivo, was also demonstrated. The acquisition of three-dimensional data sets enables subsequent analysis of a volume of the skin at cellular resolution, assimilable to a "histological block", and revealing morphological features inaccessible in conventional histology where only two-dimensional slices can be analyzed. Three-dimensional imaging also allows for different types of visualization and segmentation, which can be useful for better understanding the morphology of certain structures, revealing their sizes, shapes, three-dimensional distribution, and connections with other structures. The information provided by three-dimensional analysis could also be relevant for establishing new, more relevant quantification metrics of the skin.

The effective imaging depth in skin tissues with the LC-OCT reported here is limited by light scattering to $\sim 400 \mu \mathrm{m}$. Deeper penetration can be achieved with frequency-domain OCT (FD-OCT) devices operating at longer wavelengths (around $1300 \mathrm{~nm}$ ). These devices benefit from both higher detection sensitivity and less light scattering in tissues, but do not offer a spatial resolution as high as LC-OCT.

From a technological point of view, progress could still be made in terms of compactness and weight of the handheld probe. An approach currently being considered would be to develop an LC-OCT device with threedimensional imaging capabilities based on a Mirau interferometer rather than a Linnik interferometer $[46,62]$. The frame rate of the vertical section imaging mode is currently limited at $8 \mathrm{~Hz}$ by the mechanical response of the PZT stage to a triangle driving signal and not by camera speed or the power of the light source. The frame rate could be increased by driving the PZT stage with a sinusoidal signal and operating the camera at a variable frequency. Such a handheld LC-OCT probe with reduced weight and higher image acquisition speed would facilitate the use of the technology by dermatologists in daily practice.

In terms of applications of the technology, clinical studies are currently being conducted with the new LCOCT imaging device to demonstrate the potential in dermatology for the non-invasive diagnosis of earlystage skin cancers. 


\section{Acknowledgements}

The authors thank the whole team of engineers at DAMAE Medical, especially Olivier Levecq, Hicham Azimani, Emmanuel Cohen and Romain Allemand, for their work on the technology and design of the LCOCT prototype presented in this paper. They also thank Amyn Kassara for providing the segmentation of the three-dimensional images. They are also grateful to Anaïs Barut and David Siret as directors and managers of DAMAE Medical.

\section{References}

1. F. Fercher, “Optical coherence tomography,” J. Biomed. Opt. 1, 157-173 (1996).

2. Gh. Podoleanu, “Optical coherence tomography,” J. Microsc. 247, 209-219 (2012).

3. M. Zysk, F. T. Nguyen, A. L. Oldenburg, D. L. Marks, and S. A. Boppart, "Optical coherence tomography: a review of clinical development from bench to bedside," J. Biomed. Opt. 12, 051403 (2007).

4. J. S. Schuman, C. A. Puliafito, J. G. Fujimoto, and J. S. Duker, Optical Coherence Tomography of Ocular Diseases, 3rd ed. (Slack Inc., Thorofare, NJ, 2013).

5. H. G. Bezerra, M. A. Costa, G. Guagliumi, A. M. Rollins, and D. I. Simon, "Intracoronary optical coherence tomography: A Comprehensive Review," JACC Cardiovasc. Interv. 2, 1035-1046 (2009).

6. D. C. Adler, Y. Chen, R. Huber, J. Schmitt, J. Connolly, and J. G. Fujimoto, "Three-dimensional endomicroscopy using optical coherence tomography," Nat. Photonics 1, 709-716 (2007).

7. X. Yu et al., "Evaluating Micro-Optical Coherence Tomography as a Feasible Imaging Tool for Pancreatic Disease Diagnosis," IEEE Journal of Selected Topics in Quantum Electronic 25, 1-8 (2019).

8. J. Men, Y. Huang, J. Solanki, X. Zeng, A. Alex, J. Jerwick, Z. Zhan, R. E. Tanzi, A. Li, C. Zhou, "Optical Coherence Tomography for Brain Imaging and Developmental Biology," in IEEE Journal of Selected Topics in Quantum Electronics 22, 120-132 (2016).

9. Y. Fan, Y. Xia, X. Zhang, Y. Sun, J. Tang J, L. Zhang, H. Liao, "Optical coherence tomography for precision brain imaging, neurosurgical guidance and minimally invasive theranostics," Biosci. Trends 18, 12-23 (2018). DOI: 10.5582/bst.2017.01258

10. A. Levine, K. Wang, and O. Markowitz, “Optical coherence tomography in the diagnosis of skin cancer,” Dermatol. Clin. 35, 465-488 (2017).

11. W. Drexler, U. Morgner, F. X. Kärtner, C. Pitris, S. A. Boppart, X. D. Li, E. P. Ippen, and J. G. Fujimoto, "In vivo ultrahigh-resolution optical coherence tomography," Opt. Lett. 24, 1221-1223 (1999).

12. K. Povazay, A. Bizheva, A. Unterhuber, B. Hermann, H. Sattmann, A. F. Fercher, W. Drexler, A. Apolonski, W. J. Wadsworth, J. C. Knight, P. S. J. Russell, M. Vetterlein, and E. Scherzer, "Submicrometer axial resolution optical coherence tomography," Opt. Lett. 27, 1800-1802 (2002).

13. Y. Wang, Y. Zhao, J. S. Nelson, Z. Chen, and R. S. Windeler, "Ultrahigh-resolution optical coherence tomography by broadband continuum generation from a photonic crystal fiber," Opt. Lett. 28, 182-184 (2003).

14. A. Aguirre, N. Nishizawa, J. Fujimoto, W. Seitz, M. Lederer, and D. Kopf, "Continuum generation in a novel photonic crystal fiber for ultrahigh resolution optical coherence tomography at $800 \mathrm{~nm}$ and $1300 \mathrm{~nm}$," Opt. Express $14,1145-1160$ (2006).

15. R. A. Leitgeb, "En face optical coherence tomography: a technology review [Invited]," Biomed. Opt. Express 10, 2177-2201 (2019).

16. M. A. Choma, M. V. Sarunic, C. Yang, and J. A. Izatt, "Sensitivity advantage of swept source and Fourier domain optical coherence tomography," Opt. Express 11, 2183-2189 (2003).

17. K. S. Lee and J. P. Rolland, "Bessel beam spectral-domain high-resolution optical coherence tomography with micro-optic axicon providing extended focusing range," Opt. Lett. 33, 1696-1698 (2008).

18. R. A. Leitgeb,M. Villiger, A. H. Bachmann, L. Steinmann, and T. Lasser, "Extended focus depth for fourier domain optical coherence microscopy," Opt. Lett. 31, 2450 (2006).

19. S. Tamborski, H. C. Lyu, H. Dolezyczek, M. Malinowska, G. Wilczynski, D. Szlag, T. Lasser, M. Wojtkowski, and M. Szkulmowski, "Extended-focus optical coherence microscopy for high-resolution imaging of the murine brain," Biomed. Opt. Express 7, 4400 (2016).

20. L. Liu, K. K. Chu, G. H. Houser, B. J. Diephuis, Y. Li, E. J. Wilsterman, S. Shastry, G. Dierksen, S. E. Birket,M. Mazur, S. Byan-Parker, W. E. Grizzle, E. J. Sorscher, S. M. Rowe, and G. J. Tearney, "Method for quantitative study of airway functional microanatomy using micro-optical coherence tomography," PLoS ONE8, e54473 (2013).

21. L. Liu, C. Liu, W. C. Howe, C. J. R. Sheppard, and N. Chen, "Binary-phase spatial filter for real-time swept-source optical coherence microscopy," Opt. Lett. 32, 2375 (2007).

22. B. Yin, K. K. Chu, C.-P. Liang, K. Singh, R. Reddy, and G. J. Tearney, " $\mu O C T$ imaging using depth of focus extension by self-imaging wavefront division in a common-path fiber optic probe," Opt. Express 24, 5555 (2016). 
23. T. S. Ralston, D. L. Marks, P. S. Carney, and S. A. Boppart, "Interferometric synthetic aperture microscopy," Nat.Phys.3, 129-134 (2007).

24. S. Coquoz, A. Bouwens, P. J. Marchand, J. Extermann, and T. Lasser, "Interferometric synthetic aperture microscopy for extended focus optical coherence microscopy," Opt. Express 25, 30807 (2017).

25. L. Yu, B. Rao, J. Zhang, J. Su, Q. Wang, S. Guo, and Z. Chen, "Improved lateral resolution in optical coherence tomography by digital focusing using two-dimensional numerical diffraction method," Opt. Express 15, 7634 (2007).

26. D. J. Fechtig, A. Kumar, W. Drexler, and R. A. Leitgeb, "Full range line-field parallel swept source imaging utilizing digital refocusing," J. Mod. Opt. 62, 1801-1807 (2014).

27. J. Mo, M. de Groot, and J. F. de Boer, "Focus-extension by depth-encoded synthetic aperture in optical coherence tomography," Opt. Express 21, 10048 (2013).

28. J. Holmes, "Theory and applications of multi-beam OCT," in 1st Canterbury Workshop on Optical Coherence Tomography and Adaptive Optics, (SPIE, 2008).

29. J. Holmes, S. Hattersley, N. Stone, F. Bazant-Hegemark, and H. Barr, "Multi-channel fourier domain OCT system with superior lateral resolution for biomedical applications," in Coherence Domain Optical Methods and Optical Coherence Tomography in Biomedicine XII,J. A. Izatt, J. G. Fujimoto, and V. V. Tuchin, eds. (SPIE, 2008)

30. L. Yi, L. Sun, and W. Ding, "Multifocal spectral-domain optical coherence tomography based on bessel beam for extended imaging depth," J. Biomed. Opt. 22, 1 (2017).

31. J. Li, Y. Luo, X. Wang, N. Wang, E. Bo, S. Chen, S. Chen, S. Chen, M.-T. Tsai, and L. Liu, "Extending the depth of focus of fiber-optic optical coherence tomography using a chromatic dual-focus design," Appl. Opt. 57, 6040 (2018).

32. A. Nam, J. Ren, B. Bouma, and B. Vakoc, "Demonstration of triband multi-focal imaging with optical coherence tomography," Appl. Sci. 8, 2395 (2018).

33. R. Huber, M. Wojtkowski, J. G. Fujimoto, J. Y. Jiang, and A. E. Cable, "Three-dimensional and c-mode OCT imaging with a compact, frequency swept laser source at $1300 \mathrm{~nm}$," Opt. Express 13, 10523 (2005).

34. J. P. Rolland, P. Meemon, S. Murali, K. P. Thompson, and K. sung Lee, "Gabor-based fusion technique for optical coherence microscopy," Opt. Express 18, 3632 (2010).

35. K.-S. Lee, K. P. Thompson, P. Meemon, and J. P. Rolland, "Cellular resolution optical coherence microscopy with high acquisition speed for in-vivo human skin volumetric imaging," Opt. Lett. 36, 2221 (2011).

36. S. Liu, J. A. Mulligan, and S. G. Adie, "Volumetric optical coherence microscopy with a high space-bandwidthtime product enabled by hybrid adaptive optics," Biomed. Opt. Express 9, 3137 (2018).

37. J. M. Schmitt, S. L. Lee, and K. M. Yung, "An optical coherence microscope with enhanced resolving power in thick tissue," Opt. Commun. 142, 203-207 (1997).

38. F. Lexer, C. K. Hitzenberger, W. Drexler, S. Molebny, H. Sattmann, M. Sticker, and A. F. Fercher, "Dynamic coherent focus OCT with depth-independent transversal resolution," J. Mod. Opt. 46, 541-553 (1999).

39. Qi, P. A. Himmer, M. L. Gordon, V. X. D. Yang, D. L. Dickensheets, and I. A. Vitkin, "Dynamic focus control in high-speed optical coherence tomography based on a microelectromechanical mirror," Opt. Commun. 232, 123128 (2004)

40. A. Divetia, T.-H. Hsieh, J. Zhang, Z. Chen, M. Bachman, and G.-P. Li, "Dynamically focused optical coherence tomography for endoscopic applications,” Appl. Phys. Lett. 86, 103902 (2005).

41. V. X. D. Yang, N. Munce, J. Pekar, M. L. Gordon, S. Lo, N. E. Marcon, B. C. Wilson, and I. A. Vitkin, "Micromachined array tip for multifocus fiber-based optical coherence tomography," Opt. Lett. 29, 1754-1756 (2004).

42. A. Dubois, O. Levecq, H. Azimani, A. Davis, J. Ogien, D. Siret, and A. Barut, "Line-field confocal time-domain optical coherence tomography with dynamic focusing," Opt. Express 26, 33534-33542 (2018).

43. A. Dubois, O. Levecq, H. Azimani, D. Siret, A. Barut, M. Suppa, V. del Marmol, J. Malvehy, E. Cinotti, P. Rubegni, J.-L. Perrot, "Line-field confocal optical coherence tomography for high-resolution noninvasive imaging of skin tumors," J. Biomed. Opt. 23, 1 (2018).

44. J. Ogien, D. Siret, O. Levecq, H. Azimani, A. David, W. Xue, J. L. Perrot, and A. Dubois, "Line-field confocal optical coherence tomography," in Optical Coherence Tomography and Coherence Domain Optical Methods in Biomedicine XXIII, J. A. Izatt and J. G. Fujimoto, eds. (SPIE, 2019).

45. A. Davis, O. Levecq, H. Azimani, D. Siret, and A. Dubois, "Simultaneous dual-band line-field confocal optical coherence tomography. Application to skin imaging," Biomed. Opt. Express 10, 694-706 (2019).

46. A. Dubois, W. Xue, P. Bulkin, A.-L. Coutrot, J. Ogien, Mirau-based line-field confocal optical coherence tomography, Opt. Express, vol. 28, 918-7927 (2020).

47. J. Ogien, O. Levecq, H. Azimani, A. Dubois, "Dual-mode line-field confocal optical coherence tomography for ultrahigh-resolution vertical and horizontal section imaging of human skin in vivo," Biomed. Opt. Express 11, 1327-1335 (2020).

48. K. G. Larkin, "Efficient nonlinear algorithm for envelope detection in white light interferometry," J. Opt. Soc. Am. A 13, 832-843 (1996).

49. M. Cazalas, O. Levecq, H. Azimani, D. Siret, A. Barut, M. Suppa, V. del Marmol, J. Malvehy, E. Cinotti, P. Rubegni, J.L. Perrot, and A. Dubois, "Skin lesion imaging with line-field confocal optical coherence tomography," in Photonics in Dermatology and Plastic Surgery 2019, vol. 10851 B. Choi and H. Zeng, eds., International Society for Optics and Photonics (SPIE, 2019), pp. 61-68. 
50. G. Dejonckheere, M. Suppa, V. del Marmol, T. Meyer, and E. Stockfleth, "The actinic dysplasia syndrome diagnostic approaches defining a new concept in field carcinogenesis with multiple cSCC," J. Eur. Acad. Dermatol. Venereol. 33, 16-20 (2019).

51. M. Pedrazzani, J. Breugnot, P. Rouaud-Tinguely, M. Cazalas, A. Davis, S. Bordes, Sylvie, A. Dubois, B. Closs, "Comparison of line-field confocal optical coherence tomography images with histological sections: Validation of a new method for in vivo and non-invasive quantification of superficial dermis thickness," Skin Research and Technology 26, 398-404 (2020).

52. J. Ogien, O. Levecq, M. Cazalas, M. Suppa, V. del Marmol, J. Malvehy, E. Cinotti, P. Rubegni, J.L. Perrot and A. Dubois, "Handheld line-field confocal optical coherence tomography for dermatology," in Photonics in Dermatology and Plastic Surgery 2020, vol. 11211 B. Choi and H. Zeng, eds., International Society for Optics and Photonics (SPIE, 2020). DOI: 10.1117/12.2545546

53. J. Monnier, L. Tognetti, M. Miyamoto, M. Suppa, E. Cinotti, M. Fontaine, J. Perez, C. Orte Cano, O. Yélamos, S. Puig, A. Dubois, P. Rubegni, V. del Marmol, J. Malvehy and J.L. Perrot, "In vivo characterization of healthy human skin with a novel, non-invasive imaging technique: line-field confocal optical coherence tomography," J. Eur. Acad. Dermatol. Venereol. (2020). DOI: 10.1111/jdv.16857

54. C. Ruini, E. Sattler, "Line-Field Confocal Optical Coherence Tomography: The Golden Goose?," Aktuelle Dermatologie 46, 148-151 (2020).

55. L. Tognetti, A. Rizzo, D. Fiorani, E. Cinotti, Elisa, J.L. Perrot, P. Rubegni, "New findings in non-invasive imaging of aquagenic keratoderma: Line-field optical coherence tomography, dermoscopy and reflectance confocal microscopy, Skin Research and Technology (2020). DOI: 10.1111/srt.12882

56. C. Ruini, S. Schuh, E. Sattler, J. Welzel, "Line-field confocal optical coherence tomography - Practical applications in dermatology and comparison with established imaging methods," Skin Res. Technol. 00, 1-13 (2020). https://doi.org/10.1111/srt.12949

57. L. Tognetti, D. Fiorani, E. Cinotti, P. Rubegni, “Tridimensional skin imaging in aquagenic keratoderma: virtual histology by line-field confocal optical coherence tomography,” Int. J. Dermatol. (2020), doi:10.1111/ijd.15169

58. L. Tognetti, D. Fiorani, M. Suppa, E. Cinotti, M. Fontaine, V. Del Marmol, P. Rubegni, J.L. Perrot, "Examination of circumscribed palmar hypokeratosis with line-field confocal optical coherence tomography: Dermoscopic, ultrasonographic and histopathologic correlates," Indian J Dermatol Venereol Leprol 86:206-8 (2020)

59. L. Tognetti, A. Carraro, A. Lamberti, E. Cinotti, M. Suppa, J.L. Perrot, P. Rubegni, "Kaposi sarcoma of the glans: New findings by line field confocal optical coherence tomography examination," Skin Res. Technol. 2020. doi:10.1111/srt.12938

60. C. Ruini, S. Schuh, G. Pellacani, L. French, J. Welzel, E. Sattler, "In vivo imaging of Sarcoptes scabiei infestation using line-field confocal optical coherence tomography,” J. Eur. Acad. Dermatol. Venereol. 2020. doi:10.1111/jdv.16671

61. D. Rennie, "Nailfold Dermatoscopy in General Practice,” Aust. Fam. Physician 44, 809-812 (2015).

62. W. Xue, J. Ogien, O. Levecq, A. Dubois, "Line-field confocal optical coherence tomography based on a Mirau interferometer," SPIE's Photonics Europe, Strasbourg, France, 29 March - 2 April 2020, Proc. SPIE 11351, Unconventional Optical Imaging II, 1135110 (2020); doi.org/10.1117/12.2555691 